This item was submitted to Loughborough's Research Repository by the author.

Items in Figshare are protected by copyright, with all rights reserved, unless otherwise indicated.

\title{
Pace and critical gradient for hill runners: an analysis of race records
}

\section{PLEASE CITE THE PUBLISHED VERSION}

http://dx.doi.org/10.1515/1559-0410.1456

\section{PUBLISHER}

De Gruyter / @ American Statistical Association

\section{VERSION}

VoR (Version of Record)

\section{PUBLISHER STATEMENT}

This work is made available according to the conditions of the Creative Commons Attribution-NonCommercialNoDerivatives 4.0 International (CC BY-NC-ND 4.0) licence. Full details of this licence are available at: https://creativecommons.org/licenses/by-nc-nd/4.0/

\section{LICENCE}

CC BY-NC-ND 4.0

\section{REPOSITORY RECORD}

Kay, Anthony. 2019. "Pace and Critical Gradient for Hill Runners: An Analysis of Race Records". figshare. https://hdl.handle.net/2134/16478. 


\title{
Journal of Quantitative Analysis in Sports
}

\author{
Manuscript 1456
}

\section{Pace and Critical Gradient for Hill Runners: An Analysis of Race Records}

Anthony Kay, Loughborough University 


\title{
Pace and Critical Gradient for Hill Runners: An Analysis of Race Records
}

\author{
Anthony Kay
}

\begin{abstract}
Route choice through mountainous terrain requires a knowledge of how pace (the reciprocal of speed) varies with gradient of ascent or descent. To model this variation for runners, we analyse record times for 91 uphill and 15 downhill races or race stages. The pace is modelled as a nonlinear function of gradient and a linear function of race duration, using ordinary least squares to obtain a best fit. For the gradient-dependence, six functional forms are compared, of which a quartic is found to fit the data best; however, at steep gradients the quartic model is unrealistic and it may be argued that a linear model is more appropriate. Critical gradients, at which a runner's vertical speed (uphill or downhill) is maximised, may be calculated from a nonlinear model, although it appears that there is no uphill critical gradient within the range of our dataset.
\end{abstract}

KEYWORDS: gradient, hill running, nonlinear regression, pace, race records

Author Notes: This study would not have been possible without the efforts of the many race organisers who have made race data freely available on the internet. I would also like to thank the runners and race organisers with whom I have personally corresponded: David Eiser, Neil Shuttleworth, Chris Upson, Tim Done and David Hird.

Corresponding author: Anthony Kay, Department of Mathematical Sciences, Loughborough University, Loughborough, Leicestershire, LE11 3TU, United Kingdom.

Email: A.Kay@Lboro.ac.uk 
Kay: Hill Running Pace

\section{Introduction}

The sport of orienteering requires participants to navigate between points marked on a map, while many hill races in the United Kingdom also allow free choice of route between checkpoints at mountain summits or other prominent locations. Thus participants need to determine the fastest route between points, which will depend on the runnability and slope of the intervening terrain. Runnability is the effect of vegetation or uneven ground on running speed, and is typically classified into four categories (indicated by different shadings) on orienteering maps. Slope principally affects running speed through the work required for vertical displacement, although there must also be a contribution independent of direction, given that traversing horizontally across a steep slope is slower than running across flat ground (Arnet, 2009). Whereas the runnability shading on an orienteering map gives clear guidance to competitors on the effect of vegetation on running speed, there is no direct indication as to how their speed will depend on steepness of ascent or descent. Ideally, a competitor should know his/her pace function, a formula yielding pace $p$ as a function of gradient $m$ in the direction of travel. Pace is defined here as time per unit horizontal distance (as shown on a map), and is a more convenient variable for route choice studies than speed. In an earlier study of the route choice problem (Kay, 2012) we proposed several pace functions, derived in a rather ad hoc manner from various data sources including race results. The present work is a more careful statistical study of results from hill races over a wide range of uphill and downhill gradients, with the objective of deriving a pace function $p(m)$ that should at least be valid for a "typical" elite male hill runner. This information would also be useful to prospective organisers of new hill races who might want to estimate the winning time.

The only comparable previous analysis that we are aware of is that by Scarf (1998, 2007). However, that author used record finishing times for British fell races, which typically start and finish at the same location, and developed a model for record time as a function of total distance and total ascent. [Here and throughout this paper, "record time" or "record pace" for a hill race course refers to the fastest finishing time or pace ever recorded on that course.] He also discussed Naismith's Rule, a formula which gives time as a linear function of ascent and distance (Langmuir, 1984), again under the assumption of finishing at the same altitude as the start. If we make the further assumption that the pace at all downhill gradients is equal to that on the flat, Naismith's Rule would give pace as a function of ascent gradient, with the latter calculated as total ascent divided by total distance. However, allowing for nonlinearity of the pace-gradient relationship and relaxing the other assumptions, it is not possible to determine the pace for either ascent or descent when only the finishing time of an up-and-down hill race is given. 
An alternative to examining race results is to do controlled experiments. Townshend, Worrigham \& Stewart (2010) measured speed and oxygen uptake for eight participants running a hilly course, although the mean gradients of the uphill and downhill sections were only 0.082. Minetti et al. (2002) measured the performance of ten elite mountain runners on an inclined treadmill with gradients up to a maximum of 0.45 , both up and down, and derived the theoretical maximum running speeds at each gradient from their physiological measurements. They compared these speeds with actual speeds in small samples of uphill and downhill races and found good agreement uphill, whereas downhill race speeds were considerably slower than would be expected from physiological considerations alone. Both Minetti et al. (2002) and Townshend, Worrigham \& Stewart (2010) refer to biomechanical constraints on downhill running, and on steep downhill slopes there may also be psychological constraints (concern over personal safety). Even for uphill running, Norman (2004) and Scarf (2007) argue that treadmill experiments may not give a true representation of overground running speeds. Hence we consider that an analysis of data from races will be the most reliable basis for route choice decisions.

There are certain constraints that the pace function $p(m)$ should satisfy. First, it will be fitted to data from uphill and downhill races, but should yield a reasonable pace on level ground: we quantify this by demanding that $p(0)$ should not be faster than the current (2011) $10,000 \mathrm{~m}$ world record pace, $0.1577 \mathrm{~s}^{-1}{ }^{-1}$. The $10,000 \mathrm{~m}$ race was chosen as a suitable comparator on the basis of having similar endurance requirements to hill races: while $10,000 \mathrm{~m}$ is substantially greater than the median distance of our hill race dataset, the time of 1577 s is substantially below the median record time in the dataset.

Second, the formulation of the route choice problem by Kay (2012), based on a continuous representation of space, requires a twice-differentiable pace function (although this would not be necessary for a route choice algorithm such as that of Collischonn and Pilar (2000) which uses a discretisation of space). Third, Kay (2012) reasoned that $\mathrm{d}^{2} p / \mathrm{d} m^{2}$ should be non-negative: a route at constant gradient should never be slower than a route with the same distance and total ascent but involving some steeper and some less steep terrain. If hill runners used the same gait at all gradients, a pace function satisfying these constraints would be a reasonable expectation. However, at some uphill gradient, athletes will spontaneously make the transition from running to walking. We do not have any data on the gradient at which this occurs, and there will certainly be considerable variation between athletes, but we can expect that participants in a race will choose their gait to optimise their performance. This implies that $p(m)$ will be continuous, but not necessarily differentiable, at a gradient where the gait changes. In summary, we expect $p(m)$ to 
be a smooth function with non-negative second derivative except possibly at gaitchange gradients.

A runner's vertical speed (rate of height gain or loss) is $m / p$, which is maximised at a critical gradient where the pace function satisfies

$$
\frac{\mathrm{d} p}{\mathrm{~d} m}=\frac{p}{m}
$$

with $\mathrm{d}^{2} p / \mathrm{d}^{2}>0$; solutions of (1) may exist for $m>0$ (uphill) and/or $m<0$ (downhill). It was first noted by Davey, Hayes \& Norman (1994) that an optimal route would never ascend or descend more steeply than a critical gradient; the implications for route choice were discussed in more detail by Llobera \& Sluckin (2007) (who sought routes minimising metabolic cost rather than time taken) and by Kay (2012). Hence we shall take a particular interest in the existence and value of uphill and downhill critical gradients, denoted respectively $m_{c+}$ and $m_{c-}$, for any pace function derived from our data.

\section{Data sources}

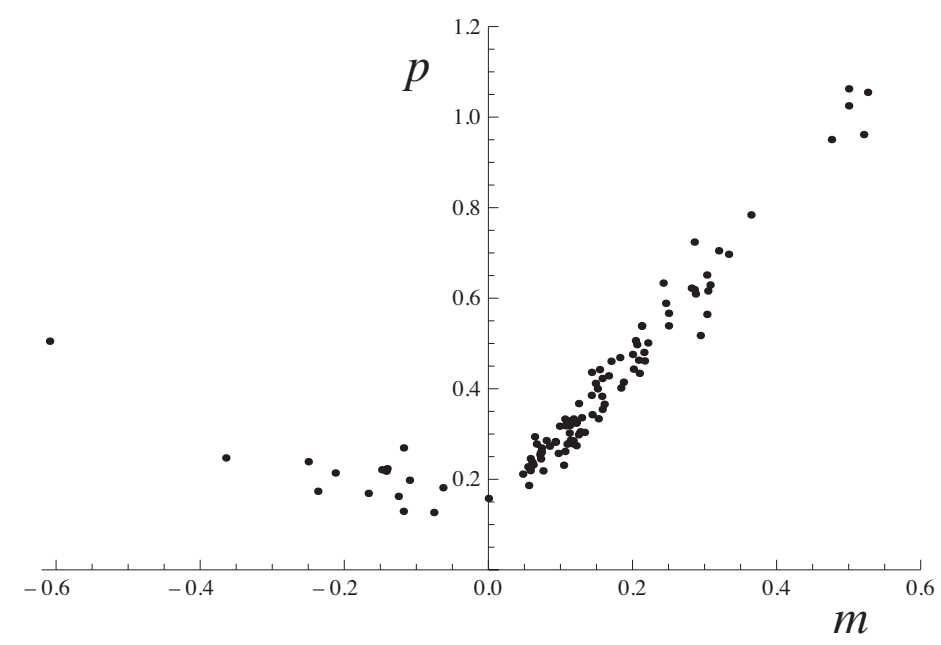

Figure 1: Record pace $p\left(\mathrm{~s} . \mathrm{m}^{-1}\right)$ vs. gradient $m$ for 91 uphill and 15 downhill races or race stages and the world $10,000 \mathrm{~m}$ track record.

All data were obtained from a search of publicly available websites conducted during 2010-12. Results from three types of race were obtained: uphill 
races, downhill races, and races including both ascent and descent, for which times for an uphill stage and/or a downhill stage were published. Races up stairs in skyscrapers, the subject of a recent study by Minetti et al. (2011), were not included. We also made a rather arbitrary decision to exclude races attaining altitudes of 4000 metres or more: races at high altitude often involve extremes of difficult terrain (including snow and ice) and climatic variation over the course, quite apart from the physiological effects of running at low atmospheric pressure. A spreadsheet listing the races, the numerical data used in this study and the URLs (not guaranteed to be stable) from which the data were obtained is available from the author.

Altogether we used record times for 91 uphill and 15 downhill races or race stages, the difference in numbers reflecting the fact that uphill races are popular, especially in continental Europe, while downhill races are rare. The mean gradient and record pace was calculated for each race or race stage, and these are plotted in Figure 1 (which also shows the $10,000 \mathrm{~m}$ world record at $m=0$ ). Mean gradients of races ranged from 0.0476 to 0.526 uphill and from -0.0631 to -0.609 downhill (observing the convention that gradients are positive uphill). The extreme downhill slope of -0.609 (from the Tryfan Downhill Dash) must be considered an influential data point, not an outlier to be ignored. There is then a gap in the data to the second steepest downhill gradient of -0.364 at the Mount Marathon Race, and then a smaller gap to the next steepest, -0.25 at the Scafell Pike Race. A significant gap also exists in the uphill data: there are five data points from Vertical Kilometre races at gradients between 0.526 and 0.476 , but the gradient of the next steepest race is 0.364. Data are also lacking at gentle gradients, both uphill and downhill. The fastest pace in the dataset is $0.127 \mathrm{~s} . \mathrm{m}^{-1}$, recorded at the Meltham Maniac Mile ( $m=-0.076$ ), while the fastest vertical speeds are $0.568 \mathrm{~m} \cdot \mathrm{s}^{-1}$ uphill at a gradient $m=0.294$ at the Grouse Grind, and $-1.474 \mathrm{~m} . \mathrm{s}^{-1}$ downhill at $m=-0.364$ at the Mount Marathon Race.

Durations of races or race stages in the dataset ranged from $300 \mathrm{~s}$ to $9160 \mathrm{~s}$ uphill and from $204 \mathrm{~s}$ to $2615 \mathrm{~s}$ downhill; our model will take account of the expected decrease in speed with increasing duration due to fatigue. However, there are many sources of variability or error that we have not been able to eliminate from our analysis of record times. First, to model pace as a function of gradient, data should ideally be taken from races or race stages at uniform gradient: no such race exists! The extent to which variability of gradient within a race introduces errors into our results depends on how nonlinear the relation between pace and gradient is. Second, we have taken no account of differences in the terrain of the races, which ranges from roads to heathery, boulder-strewn hillsides (although most are along well-trodden paths on which runnability effects will be small). Third, we have in most cases accepted without question the data on length and ascent/descent 
Kay: Hill Running Pace

given on race websites. There were a few cases in which we suspected the data to be erroneous and amended the data following a study of a map of the course. Note that errors in distance are much less serious than errors in ascent/descent, since both pace and gradient are inversely proportional to distance (so an error in distance would move a data point directly towards or away from the origin in Figure 1). In particular, the vertical descent of $609 \mathrm{~m}$ for the Tryfan Downhill Dash is known accurately from Ordnance Survey spot heights, whereas its distance of $1000 \mathrm{~m}$ is less certain; but this uncertainty will make zero difference to the linear fit proposed below for steep downhill gradients. Fourth, the races ranged from prestigious events attracting internationally leading athletes to small-scale events attracting only athletes from local clubs. We have not made any allowance for the slower times expected in the latter.

\section{Pace function models for elite hill runners}

We model our data on record pace in races as depending on gradient and race duration, where the latter is assumed to be a better determinant of fatigue than race distance (although this has not been tested). While some quite sophisticated modelling of the fatigue effect has been done (Grubb, 1998), we are only concerned to eliminate it from our consideration of gradient effects. Thus we shall follow Minetti et al. (2002) in assuming a simple linear dependence of pace on race duration,

$$
p=\tilde{p}(m)(1+k T)
$$

where the adjusted pace function $\tilde{p}(m)$ represents the dependence of pace on gradient (and is therefore the function to be used in route choice studies), and $T$ is the race duration (so that where pace and gradient data have been taken from an uphill or downhill stage of a longer race, $T$ is the duration of the entire race, not just the stage).

A variety of functional forms have been suggested for pace functions for runners and walkers. Some, such as the exponential functions of Davey, Hayes \& Norman (1994), have been proposed to model uphill pace only. Naismith's Rule and the power-law rule devised by Scarf (2007) could be used to derive pace as a function of gradient, but these rules are only intended to apply to courses with equal amounts of ascent and descent. Nevertheless, Naismith's Rule has been used for route choice problems by supposing that pace at all downhill gradients is equal to that on level ground (Scarf, 2008, Verriest, 2008, Arnet, 2009): this is equivalent to the pace function

$$
\tilde{p}= \begin{cases}p_{0} & (m<0) \\ p_{0}(1+\alpha m) & (m \geq 0)\end{cases}
$$


in which Naismith's original formulation, that a distance of 3 miles and an ascent of 2000 feet each require a time of 1 hour, yields $\alpha=7.92$ (Langmuir, 1984, Scarf, 2007). The quantity $\alpha$ gives the equivalence of distance and climb, and is also referred to as Naismith's number (Scarf, 2007). A generalisation of Naismith's Rule, to allow for the fact that running speed varies with gradient downhill as well as uphill, is the piecewise linear function

$$
\tilde{p}= \begin{cases}p_{0}\left(1+\alpha_{-} m\right) & (m<0) \\ p_{0}\left(1+\alpha_{+} m\right) & (m \geq 0)\end{cases}
$$

with $\alpha_{-}$expected to be negative. A problem with the functions (3) and (4) is that they have discontinuities in the first derivative of the pace function at $m=0$, unrelated to any change of gait. Nevertheless, another model with a discontinuity in $\mathrm{d} p / \mathrm{d} m$ has recently gained some acceptance in the anthropological research literature: this is Tobler's "Hiking function" (first published by Gorenflo \& Gale (1990) but attributed to Waldo Tobler; see also Tobler (1993), Herzog \& Posluschny (2011)), which can be re-formulated to give pace in $\mathrm{s} . \mathrm{m}^{-1}$ as

$$
\tilde{p}(m)=0.6 e^{3.5|m+0.05|} \text {. }
$$

This has a sharp minimum of pace at a downhill gradient $m=-0.05$. Kondo \& Seino (2010) suggested replacing each of the numerical coefficients in (5) with a variable, and also allowing asymmetry about the gradient of minimum pace:

$$
\tilde{p}= \begin{cases}p_{1} e^{c_{-}\left(m-m_{1}\right)} & \left(m<m_{1}\right) \\ p_{1} e^{c_{+}\left(m-m_{1}\right)} & \left(m \geq m_{1}\right),\end{cases}
$$

with $c_{-}<0<c_{+}$. This model yields critical gradients both uphill and downhill, $m_{c \pm}=1 / c_{ \pm}$. For gentle uphill gradients, where the angle of slope (in radians) is approximately equal to $m,(6)$ is similar to the exponential formulae independently derived by Davey, Hayes \& Norman (1994).

The simplest function satisfying our expectation of smoothness, with pace increasing as the gradient becomes very steep both uphill and downhill, is a quadratic as suggested by Rees (2004):

$$
\tilde{p}(m)=p_{0}\left(1+\alpha_{1} m+\alpha_{2} m^{2}\right)
$$

However, this enforces an unwarranted degree of symmetry between uphill and downhill running: specifically, uphill and downhill critical gradients will both exist and will have the same magnitude, $m_{c+}=\left|m_{c-}\right|=\alpha_{2}^{-1 / 2}$. Hence we also consider 
higher-order polynomials: cubics and quartics, as suggested respectively by Kay (2012) and Llobera \& Sluckin (2007):

$$
\tilde{p}(m)=p_{0}\left(1+\alpha_{1} m+\alpha_{2} m^{2}+\alpha_{3} m^{3}\right),
$$

and

$$
\tilde{p}(m)=p_{0}\left(1+\alpha_{1} m+\alpha_{2} m^{2}+\alpha_{3} m^{3}+\alpha_{4} m^{4}\right) .
$$

The cubic model enforces the existence of at least one of the critical gradients (uphill or downhill), while the quartic is the only one of our models that allows the existence of both, either one, or neither of the critical gradients. Other smooth functions have also been tried, but we have not been able to improve on the results shown below for polynomials.

Having chosen a functional form for $\tilde{p}(m)$, we use ordinary least squares to obtain the best fit of (2) to our data on pace. For each of the functional forms discussed above, Table 1 gives the following data (where applicable):-

- Fatigue coefficient $k$ ( \pm standard error);

- Parameter values in the model $\tilde{p}(m)$ ( \pm standard error in most cases);

- Gradient $m_{1}$ at which minimum pace (i.e. fastest horizontal speed) occurs, and the value of that minimum pace, $p_{1}$;

- Critical gradients $m_{c+}$ and $m_{c-}$ for uphill and downhill running respectively, which are the gradients at which fastest vertical speeds may be attained;

- Ratio of adjusted pace at $m=0$ to that for the world $10,000 \mathrm{~m}$ track record (denoted WR);

- Adjusted- $R^{2}$ statistic, being the most appropriate measure of the goodness of fit of the model to the data.

For each model, we can calculate the adjusted pace for each data item as

$$
\tilde{p}_{i}\left(m_{i}\right)=\frac{p_{i}}{1+k T_{i}}
$$

(in which subscript $i$ refers to $i$ 'th data item). Figure 2 shows these adjusted pace data plotted together with the fitted model function $\tilde{p}(m)$ for each of the six models. Note that units of pace and adjusted pace are s.m ${ }^{-1}$, and units of fatigue coefficient $k$ are $\mathrm{s}^{-1}$; so the parameters $k, p_{0}$ and $p_{1}$ appearing in Table 1 are dimensioned, while all others are dimensionless. 
Table 1: Model parameters \pm standard error, together with derived quantities, for six models fitted to race record pace data.

\begin{tabular}{|l||l|l|l|}
\hline Functional form & Naismith-type (3) & Piecewise linear (4) & Tobler-type (6) \\
\hline \hline$k / 10^{-5} \mathrm{~s}^{-1}$ & $2.318 \pm 1.104$ & $3.544 \pm 0.716$ & $3.559 \pm 1.057$ \\
\hline Parameters & $p_{0}=0.1413 \pm 0.0083$ & $p_{0}=0.0970 \pm 0.0057$ & $p_{1}=0.1558 \pm 0.0045$ \\
& $\alpha=10.88 \pm 0.90$ & $\alpha_{+}=17.11 \pm 1.25$ & $c_{+}=3.045 \pm 0.069$ \\
& & $\alpha_{-}=-5.575 \pm 0.641$ & $c_{-}=-2.221 \pm 0.197$ \\
& & & $m_{1}=-0.1052$ \\
\hline$m_{1}$ & [N/A] & $0[$ fixed] & -0.1052 \\
$p_{1}$ & [N/A] & $0.0970\left[\equiv p_{0}\right]$ & 0.1558 \\
\hline$m_{c+}$ & [N/A] & [N/A] & 0.3284 \\
$m_{c-}$ & [N/A] & [N/A] & -0.4503 \\
\hline$p_{0} /$ WR & 0.929 & 0.650 & 1.437 \\
\hline Adjusted $R^{2}$ & 0.9841 & 0.9941 & 0.9868 \\
\hline
\end{tabular}

\begin{tabular}{|l||l|l|l|}
\hline Functional form & Quadratic (7) & Cubic (8) & Quartic (9) \\
\hline \hline$k / 10^{-5} \mathrm{~s}^{-1}$ & $4.047 \pm 0.946$ & $4.092 \pm 0.941$ & $4.114 \pm 0.768$ \\
\hline Parameters & $p_{0}=0.1988 \pm 0.0051$ & $p_{0}=0.1938 \pm 0.0056$ & $p_{0}=0.1726 \pm 0.0053$ \\
& $\alpha_{1}=3.130 \pm 0.167$ & $\alpha_{1}=3.497 \pm 0.264$ & $\alpha_{1}=3.639 \pm 0.241$ \\
& $\alpha_{2}=9.270 \pm 0.461$ & $\alpha_{2}=9.576 \pm 0.499$ & $\alpha_{2}=17.757 \pm 1.368$ \\
& & $\alpha_{3}=-1.736 \pm 0.928$ & $\alpha_{3}=-3.100 \pm 0.877$ \\
& & & $\alpha_{4}=-23.834 \pm 3.588$ \\
\hline$m_{1}$ & -0.1688 & -0.1743 & -0.1026 \\
$p_{1}$ & 0.1463 & 0.1339 & 0.1405 \\
\hline$m_{c+}$ & 0.3285 & 0.3455 & \\
$m_{c-}$ & -0.3285 & -0.3066 & -0.2632 \\
\hline$p_{0} /$ WR & 1.341 & 1.308 & 1.165 \\
\hline Adjusted $R^{2}$ & 0.9904 & 0.9907 & 0.9940 \\
\hline
\end{tabular}


Kay: Hill Running Pace
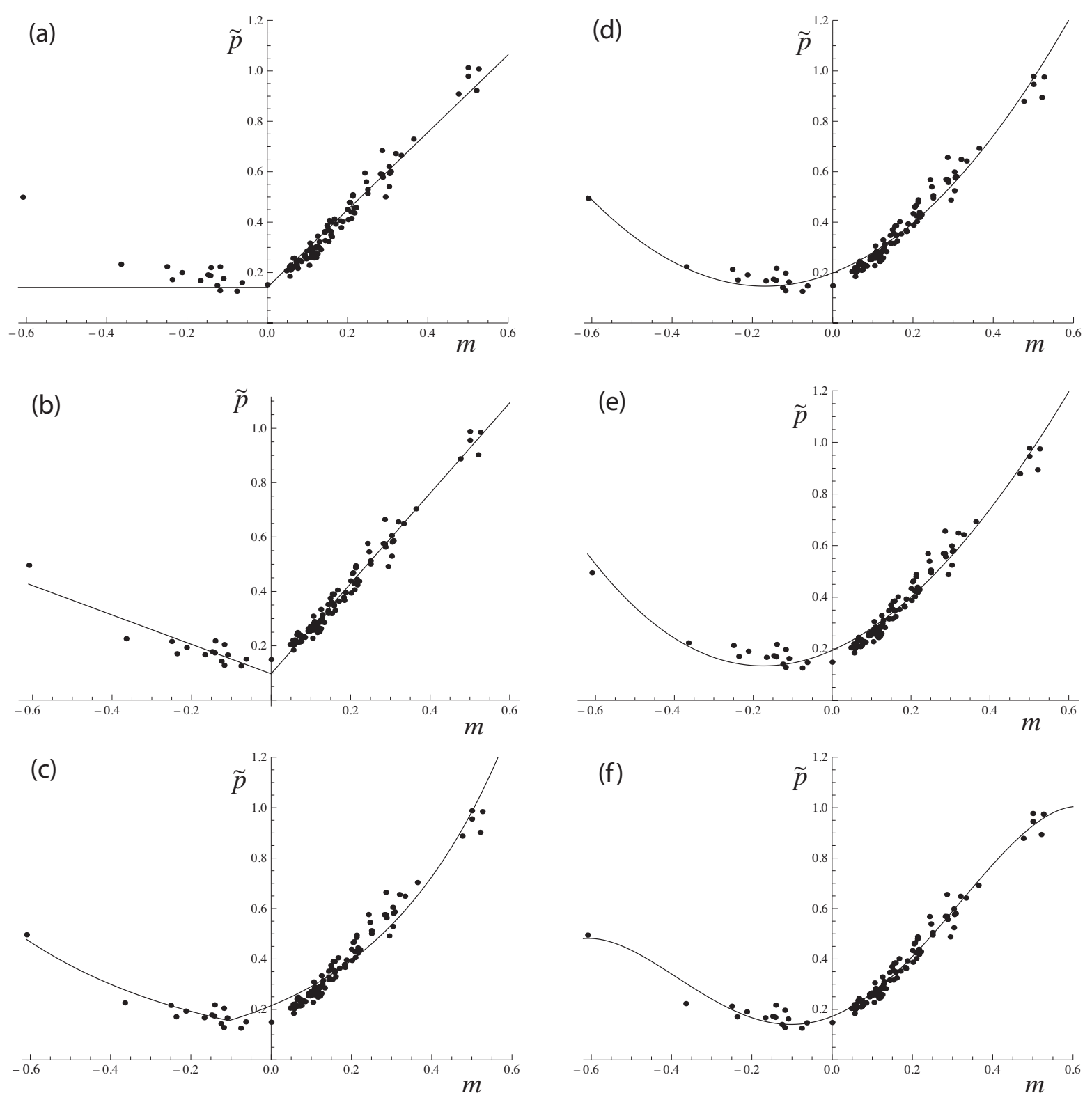

Figure 2: Adjusted pace data with various models fitted: (a) Naismith-type, (b) Piecewise linear, (c) Tobler-type, (d) Quadratic, (e) Cubic, (f) Quartic. 


\section{Discussion}

\subsection{Comparison of models}

On the basis of adjusted- $R^{2}$ values, the piecewise linear and quartic models provide the best fits to the data. However, each of these models has a serious flaw. The piecewise linear model predicts a pace $35 \%$ faster than the $10,000 \mathrm{~m}$ world record at $m=0$, so is clearly invalid at gentle gradients. The quartic model has inflection points at $m=0.3214$ and $m=-0.3864$, so the requirement that $\mathrm{d}^{2} p / \mathrm{d} m^{2} \geq 0$ is violated at uphill and downhill gradients steeper than these respective values. Thus the quartic model could still be used for route choice calculations on terrain where the slope is nowhere steeper than 0.3214 (and can be "fixed" for use on terrain steeper than this: see below), but the piecewise linear model should not be used for route choice calculations.

The Naismith-type model also predicts a faster pace than the $10,000 \mathrm{~m}$ world record at $m=0$, although in this case the world record is within the $95 \%$ confidence interval for $p_{0}$. In contrast, the Tobler-type, quadratic and cubic models all predict $p_{0}$ at least 30\% slower than the world record, and inspection of Figures 2(c), (d), (e) shows that these models also yield pace slower than the data at gentle uphill and downhill gradients. The quartic model definitely gives the best representation of pace at gentle gradients (see Figure $2(\mathrm{f})$ ), with $p_{0} / \mathrm{WR}=1.165$ being a reasonable reflection of the difference between the pace expected on a level mountain path and an athletics track.

The fastest and second-fastest paces (and adjusted paces) in our dataset are at gradients -0.076 and -0.118 respectively; only our Tobler-type and quartic models yield $m_{1}$ within the range encompassed by these values. The quadratic and cubic models predict minimum pace at much steeper gradients, close to those found by Llobera \& Sluckin (2007) and Minetti et al. (2002) to minimise metabolic energy cost. In contrast, Tobler (1993) and Kondo \& Seino (2010) find that minimum pace occurs at the gentler gradients of -0.05 and -0.07 respectively; the difference between this and our Tobler-type model can be attributed to these authors using data from walkers rather than runners. The actual values of minimum adjusted pace $p_{1}$ in all our models (except for the piecewise linear model) are slower than the two fastest paces in the dataset; this is probably due to the two fastest races both being run on roads, allowing faster running than on typical hill race terrain.

Four of our models admit critical gradients. Uphill, the quadratic, cubic and Tobler-type models all yield rather similar values of $m_{c+}$, fairly close to the value of 0.36 found by Davey, Hayes \& Norman (1994), but somewhat steeper than the 0.25 and 0.28 found respectively by Minetti et al. (2002) and Llobera \& Sluckin (2007) as being optimal for minimising metabolic energy expenditure per 
unit height gain. However, according to our quartic model there is no uphill critical gradient: ascent rate is optimised by climbing as steeply as possible. The raw data provides some support for the latter assertion: although the fastest and third fastest ascent rates in the dataset are at the moderate gradients of 0.294 and 0.303 respectively (a little lower than the estimates of $m_{c+}$ in three of our models), these were both recorded on courses on which substantial sections have steps cut into the mountainside. The advantages of steps at steep gradients are discussed by Minetti (1995), while races up skyscraper stairs often yield ascent rates considerably greater than in any mountain race (Minetti et al., 2011). Thus the stepped courses may be considered to be on atypical and advantageous terrain: removing them from the dataset, the fastest and third fastest remaining ascent rates are on the two steepest courses. Thus, if effects of differing terrain are eliminated, it appears that ascent rate is optimised by maximising steepness, at least on hills within the range of steepness in our dataset.

Downhill, the four models yield widely differing critical gradients. This is not surprising, given the dearth of data at steep downhill gradients: all four estimates of $m_{c-}$ are steeper than -0.25 , but there are only two data items at such steep downhill gradients. Minetti et al. (2002) and Llobera \& Sluckin (2007) find critical gradients for metabolic energy expenditure to be somewhat less steep downhill than uphill, with a difference of about 0.05 between $m_{c+}$ and $\left|m_{c-}\right|$, similar to that in our cubic model. Factors other than metabolism (biomechanics, psychology) would also tend to yield a less steep downhill critical gradient. On the other hand, the fastest descent rate in our dataset is on the second steepest course, at $m=-0.364$; however, this may again be accounted for by atypically advantageous terrain, with the course in this instance consisting mostly of scree, allowing very fast descent for a runner possessing the requisite technique.

\subsection{Linear approximations and Naismith's number}

The existence of a Naismith's number $\alpha$, representing the equivalence of distance and climb, assumes a linear relation between pace and gradient. However, for a nonlinear pace function Davey, Hayes \& Norman (1994) suggested that a Naismith's number could be obtained from the linear approximation about $m=0$, i.e. by setting

$$
\alpha=\frac{[\mathrm{d} p / \mathrm{d} m]_{m=0}}{p_{0}},
$$

although Norman (2004) warns that this can only be valid at gentle gradients. In fact, if the true pace function has $\mathrm{d}^{2} p / \mathrm{d}^{2} \geq 0$, (11) should give values of Naismith's number lower than those obtained from fitting linear models to data for 
positive gradients. Values of $\alpha$ from linear models will themselves depend on the range of gradients in the data and the treatment of downhill running.

Davey, Hayes \& Norman (1994) obtained $\alpha=2.8$ using (11) with an exponential model fitted to data on treadmill experiments, while applying (11) to our nonlinear models yields values (denoted $c_{+}$in our Tobler-type model and $\alpha_{1}$ in our three polynomial models) in the range $3-3.7$; this is indeed lower than any values ever obtained from linear models. The lowest of the latter is $\alpha=4.4$, obtained by Norman (2004) from a linear regression on winning times in a hilly road race with fairly gentle gradients. The classical Naismith's Rule, obtained from data for walking in Scottish mountains, has $\alpha=7.92$, while its weakly nonlinear generalisation by Scarf (2007) has $\alpha=8.6$ from British fell race data. These last three examples do not yield an estimate of uphill pace without an additional assumption being made about downhill pace; in particular, if downhill pace is assumed equal to that on flat ground, one obtains the Naismith-type model (3), for which we found $\alpha=10.88$. The above values of Naismith's number are in turn smaller than those obtained from linear models where descent is explicitly separated from ascent: this separation is partial in our piecewise linear model for which $\alpha_{+}=17.11$, while a simple linear regression on only our uphill data yields an even higher Naismith's number of 18.40 .

The wide diversity of values of Naismith's number (even before the present study) has been attributed by Norman (2004) and Scarf (2007) principally to differences in the running environment (treadmill, road or rough mountain terrain), while Kay (2012) suggested that it was related to the derivation of some values from up-and-down races while others come from experiments on uphill running. These explanations all have some validity, and sampling error must also contribute to the variation in estimates of Naismith's number, but we contend here that the fundamental problem is that a linear model is being imposed on an inherently nonlinear phenomenon. Theoretical justification for a linear model exists only at steep gradients: Minetti (1995) observes that if $|m|>0.15$ the work done in running or walking is purely positive (uphill) or purely negative (downhill), and that the efficiency is constant. Thus a constant work rate should imply a constant vertical speed, so that $p \propto m$. Fitting $\tilde{p}=b_{+} m$ to our 44 uphill data items with $m>0.15$ and $\tilde{p}=b_{-} m$ to our 6 downhill data items with $m<-0.15$, we obtain $b_{+}=1.818 \mathrm{s.m}^{-1}$ and $b_{-}=-0.8233 \mathrm{~s} . \mathrm{m}^{-1}$ with respective adjusted- $R^{2}$ values of 0.9925 and 0.9827 . For $|m|<0.15$, nonlinearity is certainly important, so a Naismith's number can only be a compromise between the true linear behaviour at steep uphill gradients and the nonlinear behaviour at gentler gradients. This compromise seems to work fairly well when predicting times taken in up-and-down fell races and mountain walks (Scarf, 2007), but yields the much smaller value of $\alpha$ found by Norman (2004) 
Kay: Hill Running Pace

from a comparatively gently graded road race, and makes the use of (11) particularly inappropriate.

\subsection{Route choice on steep gradients}

We now discuss three issues, already alluded to, that would affect the use of our pace function models for route choice on very steep hills.

First, there is some uncertainty over the existence of critical gradients. Our cubic model has an uphill critical gradient while the quartic does not; removing the steepest downhill item from the dataset yields a quartic model with no downhill critical gradient; and when cubic and quartic functions were fitted to smaller datasets while the data were being assembled, the resulting models sometimes had one or both of the critical gradients, and sometimes neither. We have concluded above that the data support the view that no uphill critical gradient exists. Minetti et al. (2002) did find optima for vertical energy cost of walking and running both uphill and downhill, but they were shallow optima. Similarly, our critical gradients, if they exist at all, will be very shallow optima for vertical speed. Thus, models with and without a critical gradient may yield substantially different route choices on steep hills, but the difference in running time will be small.

Second, the quartic model, which in most respects is the best representation of the data, has the unrealistic feature that $\mathrm{d}^{2} \tilde{p} / \mathrm{d} m^{2}<0$ at very steep gradients, both uphill and downhill. The model can be used between its inflection points, i.e. for $-0.3864 \leq m \leq 0.3214$, where $\mathrm{d}^{2} \tilde{p} / \mathrm{d} m^{2} \geq 0$, but for use outside this range of gradients we suggest adjoining other functions. With no uphill critical gradient in the quartic model, the only sensible option for $m>0.3214$ is a linear function $\tilde{p}=a+b m$ with $a$ and $b$ chosen so that $\tilde{p}$ and $\mathrm{d} \tilde{p} / \mathrm{d} m$ are continuous at $m=0.3214$; this would ensure that the straight line route is taken on slopes $m>0.3214$. Where a critical gradient does exist (downhill in the quartic model), it may be more appropriate to use the critical gradient, rather than the inflection, as the joining point for another function. This is because the linear model $\tilde{p}=b m$, which should apply at steep gradients if pace is determined purely by runner's metabolism, will join smoothly to a nonlinear curve at a critical gradient. However, the model $\tilde{p}=b m$ for $m<m_{c-}$ would make all route choices (straight or zigzag) between given points equally fast if they are everywhere steeper than the critical gradient, whereas biomechanical and psychological constraints on downhill running warrant a model that preserves the strict critical gradient property, disallowing routes that descend more steeply than $m_{c_{-}}$; a quadratic function, smoothly joined to the quartic at $m=m_{c_{-}}$, would achieve this. 
Third, the dearth of data at steep downhill gradients (with only two data items for $m<-0.25$ ) casts doubt on the reliability of any of our models where $m<$ -0.25 . To assess the reliability, we recalculated all our models with the steepest downhill item (Tryfan Downhill Dash, at $m=-0.609$ ) removed from the dataset. [We emphasise that the Tryfan data item itself is considered reliable.] The quadratic model, with its enforced symmetry, changed very little when Tryfan was omitted; but the parameters of all our other models changed substantially. While the quartic model in particular remained a very good fit to the data for $m>-0.25$, we must conclude that our models are not reliable where very steep downhill gradients $(m<$ $-0.25)$ are involved. Where a model usable for $m<-0.25$ is required, the best option would seem to be the quartic model with either a linear or quadratic function adjoined at $m=m_{c-}=-0.2632$ as described above. The linear choice, $\tilde{p}=b_{-} m$, is supported by the observation that the value $b_{-}=-0.7963 \mathrm{s.m} \mathrm{m}^{-1}$ required for a smooth join to the quartic is very close to that obtained from the linear fit to the 6 data items with $m<-0.15$, and that removing Tryfan from those 6 items also makes only a small difference to $b_{-}$. However, the participants in the Tryfan Downhill Dash are probably a self-selecting group of expert descenders, so it is not valid to infer that biomechanical and psychological constraints are unimportant: the quadratic choice may be more realistic for most runners.

\subsection{Fatigue coefficients}

Our three polynomial models all yield values of the fatigue coefficient close to $4 \times 10^{-5} \mathrm{~s}^{-1}$, with the other three models yielding somewhat smaller values. The differences are not significant: the largest value obtained minus one standard error is less than the smallest value plus one standard error. The value $k=1.773 \times$ $10^{-5} \mathrm{~s}^{-1}$ implied by the formula used by Minetti et al. (2002) may be significantly smaller; however, since the effect of fatigue is not our primary concern, we have not investigated this any further.

\section{Conclusions}

We have sought a formula to give runners' pace as a function of gradient of ascent or descent. Obviously the pace function will vary between individuals, and will also depend on the terrain. Since our calculations use record times for races, the results can be regarded as broadly applicable to elite male runners on typical hill race terrain, which consists of well made mountain footpaths in most of the continental European races which provide the majority of our data. 
A variety of functional forms have been used by previous authors to model pace or metabolic energy cost as a function of gradient. We have applied six of these functional forms to our dataset, and we conclude that on balance the quartic is the best model. It has the advantage in principle that it is the only one of the six that makes no presuppositions about the existence or otherwise of critical gradients. It provides the best fit to the data overall, and in particular yields values of $m_{1}$ (the gradient allowing maximum speed) and $p_{0}$ (pace on flat ground) consistent with the data. Its prediction of no uphill critical gradient within the range of our data is in accord with a careful examination of the individual data items with fastest vertical speeds. Observation of mountain races with free route choice shows that competitors nearly always take a direct line uphill wherever this can be done without encountering a rock face. Thus, if an optimum gradient for ascent rate does exist (as it must, since climbing a rock face is a slow process), it must be outside the range of gradients normally encountered in races - possibly at the gradient where walking gives way to scrambling, or scrambling to climbing. Given the requirements of agility and courage for steep downhill running, it is likely that a more moderate downhill critical gradient exists, with our estimate of $m_{c-} \approx-0.26$ seeming plausible but very uncertain due to the shortage of data at steep downhill gradients; indeed it is on steep descents that variations between individual runners tend to be most pronounced.

The inflection points in the quartic model do constitute a flaw that would yield unrealistic route choices on very steep gradients. We have suggested a remedy, whereby the quartic function is replaced by a linear function beyond its inflection point, or by either a linear or quadratic function beyond its critical gradient; only the quadratic would ensure that routes steeper than the critical gradient are disallowed.

Individual runners may have pace functions somewhat different from that presented here, even after multiplying by a constant factor to allow for the individual's fitness being less than that of race record holders. A runner could construct his/her own pace function from timings in a large number of mountain races, but the problems of sampling variation might be greater than in our analysis: an individual's fitness could vary considerably between races, whereas the record holder for any course must always be a runner at peak performance. However, a pace function for an individual might show clearly where the transition from walking to running occurs, whereas the change of gait has been "smoothed out" of our dataset, since it occurs at different gradients even among the select group of race record holders.

Our modelling is of doubtful utility for a runner or orienteer in the middle of a race: whereas a simpler formula such as Naismith's Rule could be used to estimate timings on the run, route choice calculations with a nonlinear pace model would not be feasible in that situation. However, knowledge of one's personal critical gradient(s) could obviate wasted time on steep slopes during a race. A nonlinear 
model could be used by competitors to plan routes (using a route choice algorithm) or estimate timings where a course is known in advance (i.e. in mountain races, but not orienteering competitions), although even then terrain runnability may have as much or more influence on route choice than topography. While orienteers are usually concerned with the hindrance to running provided by soft, uneven, or thickly vegetated terrain, we have noted that stepped paths can be an aid to running speed on steep uphill slopes.

Finally, we note that this is probably the most thorough study so far of overground running pace as a function of gradient. By comparing with previous studies of metabolic energy cost of gradient running, it should be possible to identify the extent to which non-physiological factors affect mountain running pace.

\section{References}

Arnet, F. 2009. "Arithmetical route analysis with examples of the long final courses of the World Orienteering Championships 2003 in Switzerland and 2005 in Japan." Scientific Journal of Orienteering 17: 4-21.

Collischonn, W. and J.V. Pilar. 2000. "A direction dependent least-cost-path algorithm for roads and canals." International Journal of Geographical Information Science 14: 397406.

Davey, R.C., M. Hayes and J.M. Norman. 1994. "Running uphill: an experimental result and its applications." Journal of the Operational Research Society 45: 25-29.

Gorenflo, L.J. and N. Gale. 1990. "Mapping regional settlement in information space." Journal of Anthropological Archaeology 9: 240-274.

Grubb, H.J. 1998. "Models for comparing athletic performance.” The Statistician 47: 509521.

Hayes, M. and J.M. Norman. 1984 "Dynamic programming in orienteering: route choice and the siting of controls." Journal of the Operational Research Society 35: 791-796.

Herzog, I. and A. Posluschny. 2011. "Tilt - Slope-dependent least cost path calculations revisited. In: On the Road to Reconstructing the Past. Computer Applications and Quantitative Methods in Archaeology: Proceedings of the 36th International Conference, edited by Erszébet Jerem, Ferenc Redô and Vajk Szeverényi, 236-242. Budapest: Archaeolingua Foundation.

Kay, A. 2012. "Route choice in hilly terrain." Geographical Analysis 44: 87-108.

Kondo, Y. and Y. Seino. 2010. "GPS-aided walking experiments and data-driven travel cost modeling on the historical road of Nakasendō-Kisoji (Central Highland Japan)." In: Making History Interactive. Computer Applications and Quantitative Methods in Archaeology. Proceedings of the 37th International Conference, 158-165, edited by Bernard Frischer, Jane W. Crawford and David Koller. Oxford: Archaeopress.

Langmuir, E. 1984. Mountaincraft and Leadership. Edinburgh: The Scottish Sports Council. 
Kay: Hill Running Pace

Llobera, M. and T.J. Sluckin. 2007. "Zigzagging: Theoretical insights on climbing strategies." Journal of Theoretical Biology 249: 206-217.

Minetti, A.E. 1995. "Optimum gradient of mountain paths." Journal of Applied Physiology 79: $1698-1703$.

Minetti, A.E., C. Moia, G.S. Roi, D. Susta, and G. Ferretti. 2002. "Energy cost of walking and running at extreme uphill and downhill slopes." Journal of Applied Physiology 93: 1039-1046.

Minetti, A E., D. Cazzola, E. Seminati, M. Giacometti, and G.S. Roi. 2011. "Skyscraper running: physiological and biomechanical profile of a novel sport activity." Scandinavian Journal of Medicine and Science in Sports 21: 293-301.

Norman J.M. 2004. "Running uphill: energy needs and Naismith's rule.” Journal of the Operational Research Society 55: 308-311.

Rees, W.G. 2004. "Least-cost paths in mountainous terrain." Computers and Geosciences 30: 203-209.

Scarf, P. 1998. "An empirical basis for Naismith's rule." Mathematics Today 34: 149-151.

Scarf, P. 2007. Route choice in mountain navigation, Naismith's rule, and the equivalence of distance and climb. Journal of Sports Sciences 25: 719-726.

Scarf, P. 2008. "A mathematical excursion in the isochronic hills." Mathematics Today 44: 163-167.

Townshend, A.D., C.J. Worringham and I.B. Stewart. 2010. "Spontaneous pacing during overground hill running." Medicine and Science in Sports and Exercise 42: 160-169.

Tobler, W. 1993. "Non-isotropic geographic modelling." Technical Report 93-1, National Center for Geographic Information and Analysis, Santa Barbara, CA. Accessed 3 October 2012. http://www.geodyssey.com/papers/tobler93.html .

Verriest, E.I. 2008. "A variant to Naismith's problem with application to path planning." In Proceedings of the 17th World Congress, International Federation of Automatic Control, 7136-7141, edited by Myung Jin Chung and Pradeep Misra. Oxford: Elsevier. 